\title{
Efficient method for calculating the transmission coefficient of two-dimensional quantum wire structures
}

\author{
Chinghong Yiu and Jian Wang \\ Department of Physics, The University of Hong Kong, Pokfulam Road, Hong Kong
}

(Received 15 April 1996; accepted for publication 2 July 1996)

\begin{abstract}
We present a very simple and efficient method for calculating the transmission coefficient of two-dimensional quantum wire structures based on the time-dependent solution of the Schrödinger equation. We apply the new method to a specific two-dimensional quantum wire structure. The new method is much faster than the finite element method and can be used to study electron transport in the presence of electron-phonon interaction and nonlinear interactions in the Schrödinger equation.

(C) 1996 American Institute of Physics. [S0021-8979(96)06319-0]
\end{abstract}

There has been considerable interest recently in the properties of ballistic electron transport in confined semiconductor geometries. ${ }^{1}$ This is due partly to the potential applications to ultrafast electronic devices. Indeed, devices with operation principles entirely based on quantum interference have been proposed and fabricated. ${ }^{2,3}$ On the theory side, a thorough understanding of quantum transport including the ballistic regime is of great importance. So far, much theoretical effort has focused on analyzing the transmission pattern of electrons propagating through various ultrasmall one- and two-dimensional quantum systems where electron waves maintain phase coherence. A particularly interesting system is the T-shaped quantum wire investigated by Sols et al. ${ }^{4}$ as they argued that such a structure can operate as a transistor. Another interesting structure is the electron wave guide coupler. $^{5}$ This is an electronic analog of the optical directional coupler and it switches electrons from one quantum wire to another. In these quantum devices, switching is provided not by tuning a current but, rather, by tuning the phase of the electron waves. In order to study the quantum transport of two-dimensional devices, one must deal with open systems where a scattering problem of charge carriers by some peculiar boundary must be solved.

Currently, there are several methods to calculate the transmission coefficient including (1) the mode matching method $^{6}$ which is very fast but only suitable for regular shaped two-dimensional quantum wire structures with constant potential energy; (2) the finite element method, ${ }^{7,8}$ although much slower, can handle quantum wire structure of arbitrary shape and potential; and (3) the recursive Green function method, ${ }^{4}$ whose speed is comparable to that of the finite element method and can treat the structure of any shape and potential. However, the recursive Green function method does not provide the electronic wave function inside the quantum wire structure that is essential in studying the physical properties such as dwell time and dynamic conductance. ${ }^{9}$ These three methods are static and time independent. Another method currently in use is the timedependent method ${ }^{10-13}$ which solves the time-dependent Schrödinger equation numerically and calculates the transmission coefficient based on the transmitted wave. The advantage of this method is that it can deal with the system of any shape or potential and the computational time scales linearly with the system size. More important, it can be used to study the dynamical behavior in the presence of electronphonon interaction, ${ }^{14,15}$ the nonlinear interactions in the Schrödinger equation, ${ }^{16}$ the self-consistent solution of the two-dimensional quantum wire structure including many electron effects, ${ }^{17}$ and the strongly correlated quantum system. ${ }^{18}$ In order to facilitate the time-dependent calculation, one has to prepare an initial normalized Gaussian wave packet with an average energy $E_{0}$ and width $\Delta E$ which depends on the spatial spread $\sigma$ of the wave packet, follow the time evolution of the wave packet, and then collect the transmitted wave $\Psi_{T}$ which is identified as the transmission coefficient $T\left(E_{0}, \sigma\right)=\int\left|\Psi_{T}\right|^{2} d \Omega$. To obtain the transmission coefficient $T$ as a function of incident electron energy $E$, one must repeat the calculation for many different incident energies $E_{0}$. The transmission coefficient $T\left(E_{0}, \sigma\right)$ calculated this way depends on the spatial spread of the wave packet. Since the Gaussian wave packet is a superposition of many plane waves, the transmission coefficient $T\left(E_{0}, \sigma\right)$ is only an approximation of the exact transmission coefficient. To obtain a more accurate result, one has to increase the spatial spread of the wave packet $\sigma$ so that it is composed of fewer plane waves. In the large $\sigma$ limit, one recovers the exact transmission coefficient $T(E)$. The tradeoff is that the system size, and hence the computational time, is increased, which makes this method disadvantage over the static method. In this communication, we present a more efficient method based the time-dependent method described above. The improvement of our method is twofold: (1) the transmission coefficient calculated using the new method is exact, and (2) one needs only one time-dependent calculation at a particular $E_{0}$ to get the whole $T(E)$ versus $E$ curve which makes this method very efficient. This is because when performing the time-dependent calculation for a wave packet at an average incident electron energy $E_{0}$, the transmitted wave has much more information than merely $T\left(E_{0}\right)$. Actually, the entire $T(E)$ versus $E$ curve can be extracted from the transmitted wave, which we will describe below.

To illustrate our method, let us consider a onedimensional scattering problem, where a potential barrier is located in the region $(0, a)$. The scattering wave function for an incident plane wave with wave vector $k$ can be written as

$$
\psi=\left\{\begin{array}{l}
e^{i k x}+r(k) e^{-i k x} \quad x<0 \\
t(k) e^{i k x} \quad x>a
\end{array},\right.
$$




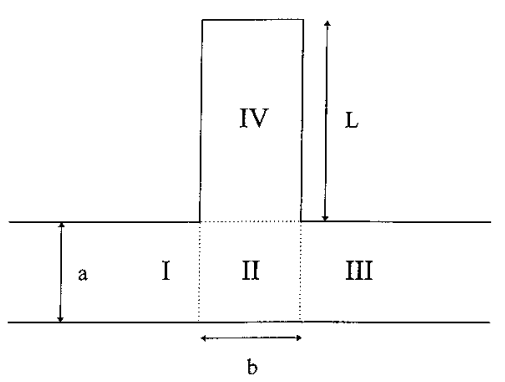

FIG. 1. Schematic view of the T junction where regions II and IV form the interaction region. The width of the quantum wire $a$ and the width of the side arm $b$ are taken to be $100 \AA$. The height of the side arm $L$ is $200 \AA$.

where $t(k) \exp (i k x)$ is the transmitted wave and $T=|t(k)|^{2}$ is the transmission coefficient. For an incident Gaussian wave packet, which is a superposition of plane waves, the initial wave function is given by

$$
\begin{aligned}
\psi(x, t=0) & =\frac{1}{\left(\pi \sigma^{2}\right)^{1 / 4}} e^{i k_{0} x} e^{-\left(x-x_{0}\right)^{2} / 2 \sigma^{2}} \\
& =\sum_{k} f(k) e^{i k x},
\end{aligned}
$$

where

$$
f(k) \sim e^{-\sigma^{2}\left(k-k_{0}\right)^{2} / 2}
$$

is the Fourier transform of $\psi(x, t=0)$. After the wave completely gets out of the scattering region $(0, a)$, the transmitted wave is given by ${ }^{19,20}$

$$
\psi_{T}(x, t)=\sum_{k} t(k) f(k) e^{i k x-i \omega t},
$$

where $\omega=\hbar k^{2} / 2 m, t$ is the time, and $t(k)$ is the transmission amplitude which can be calculated by Fourier transform of $\psi_{T}$. Our method proceeds as follows: we follow the timeevolution of a Gaussian wave packet, compute the transmitted wave, and finally the transmission amplitude is calculated by Fourier transform:

$$
t(k) f(k)=\psi_{T}(k)
$$

where $\psi_{T}(k)$ is the Fourier transform of $\psi_{T}(x, t)$ and $f(k)$ is given by Eq. (2). We can also calculate the reflection coefficient from the reflected wave in a similar manner.

To demonstrate the efficiency of our method we consider a two-dimensional quantum structure $\left(\mathrm{T}\right.$-junction $\left.{ }^{4}\right)$ shown in Fig. 1. Assuming that the electron coherence length is longer than the size of the junction, we treat the transport of electrons ballistically within the structure, but include scattering of the electrons due to the presence of the junction. Such scattering, due to geometric junctions in two dimensions, affects transmission much more significantly than in one dimension. The quantum wires are modeled in a similar fashion to those of Sols et al. ${ }^{4} \mathrm{We}$ assume, for simplicity of the calculation, that the boundaries are hard walls.

The time evolution of the electron wave function is governed by the time-dependent Schrödinger equation written as

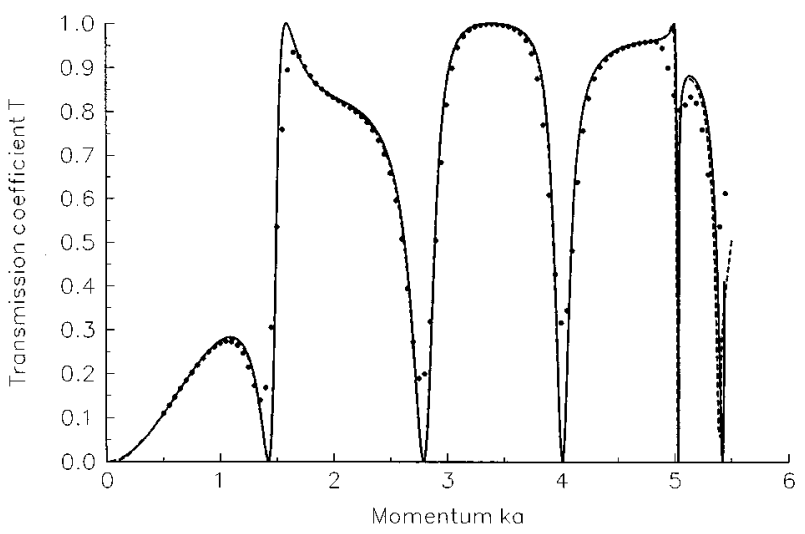

FIG. 2. The transmission coefficient of the $\mathrm{T}$ junction vs incident energy. The solid line corresponds to the exact solution using the mode matching method and the solid circle to the approximate solution using the timedependent method where $\sigma=1000 \AA$ and 100 Gaussian wave packets with different $k_{0}$ were used. The dashed line is obtained using our method where we have used $k_{0}=3.0$ and $\sigma=100 \AA$.

$$
\begin{aligned}
i \hbar \frac{\partial \psi(x, y, t)}{\partial t}= & -\frac{\hbar^{2}}{2 m^{*}}\left(\frac{\partial^{2} \psi(x, y, t)}{\partial x^{2}}+\frac{\partial^{2} \psi(x, y, t)}{\partial y^{2}}\right) \\
& +V(x, y) \psi(x, y, t) .
\end{aligned}
$$

We choose the initial condition to be a Gaussian wave packet in the first subband located inside lead $I$ :

$$
\begin{aligned}
& \psi(x, y, t=0) \\
& =\frac{1}{\left(\pi \sigma^{2}\right)^{1 / 4}} e^{i k_{0} x} e^{-\left(x-x_{0}\right)^{2} / 2 \sigma^{2}} \frac{1}{(2 a)^{1 / 2}} \sin \left(\frac{\pi}{a} y\right),
\end{aligned}
$$

where $\hbar k_{0}$ gives the initial average momentum [the initial average energy is $\left.E_{0}=\hbar^{2}\left(k_{0}^{2}+\pi^{2} / a^{2}\right) / 2 m^{*}\right], x_{0}$ gives the location of the peak at $t=0, \sigma$ determines the spatial spread of the wave packet and gives an energy width $\Delta E$ $=\sqrt{2 \hbar^{2} E_{0} / m^{*}}(1 / \sigma)$, and $m^{*}=0.067 m_{0}$ is the isotropic effective mass for gallium arsenide. Equation (2) is solved using a typical Crank-Nicholson type algorithm ${ }^{21-23}$ on a $10000 \times 20$ grid with a typical spatial mesh size of $5 \AA$ and a time step less than $0.23 \mathrm{fs}$ in our calculation.

The exact transmission coefficient $T(E)$ for the $\mathrm{T}$ junction can be calculated using the mode-matching method and is depicted in Fig. 2 (solid line). The approximate transmission coefficient $T(E, \sigma)$, obtained by sending a number of Gaussian wave packets and collecting the transmitted wave, is also plotted in Fig. 2 for comparison (solid circle) where we have chosen a large spatial spread $\sigma=1000 \AA$ and the width of the lead is $a=100 \AA$. In this calculation, we have used 100 Gaussian wave packets with different values of $k_{0}$ uniformly distributed between the first and the second subbands. We see that because of the large $\sigma$ the agreement is very good except at the resonance (reflection peak). ${ }^{24}$ In Fig. 2 we plot $T(E)$ versus incident energy $E$ using our method (dashed line), where we have used only one Gaussian wave packet with the average momentum $k_{0}=3.0$, the width of the lead $a=100 \AA$, and the spatial spread of the wave packet $\sigma=100 \AA$. It agrees with the exact result (solid line in Fig. 2) very well. The agreement will be better if we increase the 
system size. A practical way of getting an accurate transmission coefficient curve $T(E)$ vs $E$ is to do not just one but several calculations with different average momentum $k_{0}$ so that each $k_{0}$ will cover a portion of the curve and overlap with the other $k_{0}$. We have generated the entire curve using several different $k_{0}$. Similar results are obtained using different parameters such as $k_{0}$ and $\sigma$. We have also applied our method to a quantum billiard and a multilead structure successfully. In these calculations, we typically need to iterate about 7000 time steps in solving the time-dependent Schrödinger equation. All the calculations are done on an IBM RISC 6000 machine. It usually takes several hours for each calculation, which is much faster than the finite element method.

There are several ways to improve the present method. First of all, we can use the absorbing boundary condition ${ }^{11}$ for the reflected wave so that the numerical grid size can be reduced. Second, instead of using the tight-binding version of Eq. (5) which is accurate up to second order in the mesh width $\delta$ which is dimensionless, we can use the fast Fourier transform method to deal with the kinetic energy ${ }^{25}$ and eliminate the error from $\delta$.

In summary, we have presented a simple and efficient method to calculate the transmission coefficient for twodimensional quantum wires based on the time-dependent solution of Schrödinger equation. Currently, this method is being extended to include the magnetic field.

This work was supported by a RGC research grant from the Hong Kong government under Grant No. HKU 261/95P and a grant from Hung Hing Ying Physical Science Research Fund. The authors thank Qingrong Zheng for useful discussions. They also thank the computer center of The University of Hong Kong for computational facilities.
${ }^{1}$ See, for example, articles in Nanostructure Physics and Fabrication, edited by M. A. Reed and W. P. Kirk (Academic, New York, 1989).

${ }^{2}$ F. Capasso, S. Sen, F. Beltram, L. M. Lunardi, A. S. Vengurlekar, P. R. Smith, N. J. Shah, R. J. Malik, and A. Y. Cho, IEEE Trans. Electron Devices 36, 2065 (1989).

${ }^{3}$ S. Datta, Superlattices Microstruct. 6, 83 (1989).

${ }^{4}$ F. Sols, M. Macucci, U. Ravaioli, and K. Hess, Appl. Phys. Lett. 54, 350 (1989)

${ }^{5}$ J. A. del Alamo and C. C. Eugster, Appl. Phys. Lett. 56, 78 (1990); N. Tsukada, A. D. Wieck, and K. Ploog, Appl. Phys. Lett. 56, 2527 (1990); J. Wang, H. Guo, and R. Harris, Appl. Phys. Lett. 57, 3075 (1991); J. Wang, Y. J. Wang, and H. Guo, Phys. Rev. B 46, 2420 (1992).

${ }^{6}$ R. L. Schult, D. G. Ravenhall, and H. W. Wyld, Phys. Rev. B 39, 5476 (1989).

${ }^{7}$ C. S. Lent and D. J. Kirkner, J. Appl. Phys. 67, 6353 (1990).

${ }^{8}$ Y. J. Wang, J. Wang, and H. Guo, Phys. Rev. B 49, 1928 (1994).

${ }^{9}$ M. Büttiker, Phys. Rev. B 27, 6178 (1983); M. Büttiker, A. Pretre, and H. Thomas, Phys. Rev. Lett. 70, 4114 (1993).

${ }^{10}$ H. Guo, K. Diff, G. Neofotistos, and J. D. Gunton, Appl. Phys. Lett. 53, 131 (1988).

${ }^{11}$ For switching by voltage in T-shaped structure, see, for example, L. F. Register, U. Ravaioli, and K. Hess, J. Appl. Phys. 69, 7153 (1991).

${ }^{12}$ J. Wang and H. Guo, Phys. Rev. B 48, 12072 (1993).

${ }^{13}$ H. De Raedt, Comput. Phys. Rep. 7, 1 (1987).

${ }^{14}$ J. M. Mohaidat, K. Shum, and R. R. Alfano, Phys. Rev. B 48, 8809 (1993).

${ }^{15}$ L. F. Register and K. Hess, Phys. Rev. B 49, 1900 (1994).

${ }^{16}$ G. Jona-Lasinio, C. Presilla, and F. Capasso, Phys. Rev. Lett. 68, 2269 (1992).

${ }^{17}$ J. Wang and C. H. Yiu (unpublished).

${ }^{18}$ P. de Vries and H. De Raedt, Phys. Rev. B 47, 7929 (1993)

${ }^{19}$ S. Collins, D. Lowe, and J. R. Barker, J. Phys. C 20, 6233 (1987).

${ }^{20} \mathrm{R}$. Shankar, Principles of Quantum Mechanics (Plenum, New York, 1980), p. 181.

${ }^{21}$ I. Galbraith, Y. S. Ching, and E. Abraham, Am. J. Phys. 52, 60 (1984).

${ }^{22}$ M. Cahay, J. P. Kreskovsky, and H. L. Grubin, Solid-State Electron. 32, 1185 (1989).

${ }^{23}$ F. Sols, M. Macucci, U. Ravaioli, and K. Hess, J. Appl. Phys. 66, 3892 (1989)

${ }^{24}$ J. Wang, Y. J. Wang, and H. Guo, Appl. Phys. Lett. 65, 1793 (1993).

${ }^{25}$ D. Kosloff and R. Kosloff, J. Comput. Phys. 52, 35 (1983). 